REVISTA

MEXICANA DE

ECONOMÍA Y

FINANZAS

REMEF

(THE MEXICAN JOLRNAL OF
ECONOMICS AND FINANCE)
Revista Mexicana de Economía y Finanzas, Nueva Época

Volumen 16 Número 3, Julio - Septiembre 2021, pp. 1-20, e645

THE ECONOMICS AND FINANCE EFFECTS OF THE COVID-19 PANDEMIC Editor: Dr. Ignacio Perrotini

DOI: https://doi.org/10.21919/remef.v16i3.645

(Recibido: 29/marzo/2021, aceptado: 7/julio/2021, publicado: 7/julio/2021)

\title{
Efectos de la Covid-19 y la restricción a la movilidad de las personas sobre el consumo adictivo de cigarros en México, 2005-2020
}

\author{
Miguel Ángel Mendoza-González ${ }^{1}$ - Universidad Nacional Autónoma de México, México \\ Alberto Villagra-Piña - Universidad Nacional Autónoma de México, México
}

El objetivo de este artículo es analizar el impacto que han tenido la Covid-19 y la restricción de movilidad de las personas en el consumo de cigarrillos en México. La metodología son modelos de cointegración fmols con endogeneidad y cambio estructural para identificar comportamientos miopes o racionales de acuerdo con la Teoría de Adicción Racional. Los resultados muestran que los precios y eventos estresantes son el principal determinante del consumo de cigarros. Se recomienda incidir significativamente sobre los precios del tabaco. Las implicaciones son que el distanciamiento social y la restricción a la movilidad de las personas han incrementado el consumo de cigarros, el alcance limita la inclusión de otros factores como la pérdida del empleo. La originalidad del artículo radica en que en estudios previos no se han considerado eventos estresantes como la Covid-19. La principal conclusión es que el aumento de precios relativos de cigarros previo a las condiciones de pandemia y el choque de ingreso son la principal causa de la reducción de las ventas de cajetillas de cigarros durante la crisis sanitaria y económica de 2020.

Clasificación JEL: D42, H22, I10, I12.

Palabras clave: Covid-19, restricción a la movilidad de personas, consumo adictivo de cigarros, elasticidad precio, choque de ingreso, México, adicción racional.

\section{Effects of Covid-19 and people mobility constraint on addictive consumption of cigarettes in Mexico, 2005 - 2020}

The aim of this research paper is to analyze the impact of Covid-19 and the restriction of the mobility of people on cigarette consumption in Mexico. For this purpose, fmols cointegration models with endogeneity and structural change are used to identify myopic or rational behaviors in accordance with the Rational Addiction Theory. The results show that the prices and stressful events are the main determinant of cigarette consumption. It is recommended to regulate the tobacco prices. This implies that social distancing and the restriction of the mobility of people have increased consumption of cigarettes. The scope of this research limits to consider other variables like the job loss. The originality of this work is to take in consideration stressful events like Covid-19. The main conclusion is that the increase in the relative prices of cigarettes prior to pandemic conditions and the income shock are the leading cause of the reduction in sales of cigarette packs during the health and economic crisis in 2020. JEL Classification: D42, H22, I10, I12.

Keywords: Covid-19, People mobility constraints, addictive consumption of cigarettes, price elasticity, income shock, Mexico, rational addiction.

${ }_{1}^{1}$ Autor de correspondencia. División de Posgrado de la Facultad de Economía de la Universidad Nacional Autónoma de México, Ciudad Universitaria, Edificio de Posgrado, cubículo 213, segundo piso, C.P. 10450, Delegación Coyoacán, Ciudad de México, México, Teléfono: + 52 (55) 56-22-18-88 ext. 4889. E-mail: mendozag@unam.mx

*Esta investigación tuvo el apoyo del proyecto financiado por UNAM: PAPIIT-IN308721 “Políticas públicas de reactivación y reestructuración económica urbana en México ante impactos económicos y sociales de la COVID-19 en México”. 


\section{Introducción}

En la mayoría de los países de ingreso medio y bajo (LMICs por sus siglas en inglés) existe una tendencia creciente en el consumo de cigarros y en el consecuente aumento de muertes prematuras por el cáncer de pulmón (WHO, 2019). El 80\% de los fumadores viven en LAMICs y, aunque, la prevalencia a fumar se ha reducido a nivel mundial no es suficiente para alcanzar la meta de una reducción del 30\% hacia el 2025 (WHO, 2019). En el caso de México la prevalencia a fumar es de $17.9 \%$ en 14.8 millones de adultos y $5.7 \%$ en 1.3 millones de adolescentes que suman 16.1 millones de fumadores (INEGI-INSP, 2018) y es menor a los 17.3 millones de fumadores de 2011 (INSPINPRFM, 2011). Sin embargo, con datos sobre el volumen de ventas de cajetillas de cigarros identificamos una tendencia contraria a la prevalencia a fumar donde el consumo mensual de cajetillas por persona fumadora de 7.5 en 2011 pasó a 14.2 en 2018, lo cual indica que el consumo adictivo de cigarros aumentó $89 \%$ en tan sólo siete años.

En las discusiones sobre Políticas Públicas para reducir el consumo de cigarros se menciona que el mejor mecanismo económico es el fiscal que consiste en aumentar el precio de los cigarros con la aplicación de impuestos. Para entender el proceso de una política impositiva se considera la teoría económica convencional sobre la elección de los consumidores que sostiene que los fumadores son sensibles a los cambios de los precios como cualquier otro bien (Mas-Colell, Whinston, \& Green, 1995). En cambio, en el enfoque alternativo sobre el consumo adictivo se supone un comportamiento de elección miope de los fumadores cuando se considera el consumo inmediato pasado o en el marco analítico de elección racional en la que las decisiones de compra de cigarros involucran tanto el consumo pasado como el consumo futuro (Becker \& Murphy, 1988). En estos enfoques económicos alternativos sobre las adicciones, el fumador toma en cuenta en sus decisiones el precio de los cigarros y posibles costos adicionales como la aplicación de nuevos impuestos, gastos médicos o costos intangibles como la desaprobación social (Gerrero, Muñoz, Sáenz de Miera, \& Reynales, 2013; Hall, Fleischer, Reynales, Arillo, \& Trasher, 2015; Liu, Rizzo, \& Sun, 2015; WB, 2019). Todo ello tiene como consecuencia una mayor sensibilidad (elasticidad) de los consumidores de cigarros a cambios de los precios (Becker, Grossman, \& Murphy, 1991).

En gran parte de la literatura se analiza si los consumidores de cigarros se comportan de acuerdo con la teoría económica de las adicciones donde son más sensibles a los cambios de los precios relativos de los cigarros que a otras condiciones de la demanda. Diversos estudios han mostrado resultados heterogéneos en cuanto a la sensibilidad (elasticidad) del consumo de cigarros a los cambios de sus precios relativos. Algunos confirman el modelo miope de adicción con valores de la elasticidad entre -0.2 y -0.9 (Adenji, 2019; Gjika, Zhllima, Rama, \& Imami, 2020; Guindon, Paraje, \& Chaloupka, 2018; Olivera, Cermeño, Sáenz de Miera, Jiménez, \& Reynales, 2010; CEFP, 2018; Bardsley \& Olekalns, 1999; Liu, Rizzo, \& Sun, 2015) dependiendo de la fuente de los datos utilizados, el periodo y el método de cálculo. Algunos otros confirman la existencia de estados estacionarios inestables con valores de la elasticidad precio de la demanda en el largo plazo superior a la unidad (Grossman \& Chaloupka, 1998; Ferguson, 2000; Iwasaki, Tremblay, \& Horton, 2006; Guindon, Paraje, \& Chaloupka, 2018; Jiménez, Sáenz de Miera, Reynales, Water, \& Hernández, 2008). Y pocos han encontrado comportamientos muy elásticos del consumo de cigarros superiores a dos en términos 
absolutos (Olivera, Cermeño, Sáenz de Miera, Jiménez, \& Reynales, 2010). Todo ello comprueba la Teoría de Adicción Racional tal como lo menciona Baltagi (2007) en su revisión de metaestudios.

En esta investigación se aporta a la discusión sobre el consumo adictivo de cigarros en dos direcciones: en la primera se discute si la medición del consumo de cigarros con las ventas mensuales de cajetillas por fumador implica el comportamiento adictivo de los fumadores miopes o racionales de acuerdo a la teoría económica de las adicciones en el periodo previo a la pandemia de la Covid-19 en México; y, si las medidas para reducir el contagio del virus, como son el distanciamiento social y las restricciones a la movilidad de las personas, se vinculan con el aumento del consumo adictivo de cigarros durante la crisis económica de México en 2020. Para ello se utilizan modelos econométricos de consumo de cigarros de acuerdo el análisis de cointegración, con endogeneidad y cambio estructural en el contexto de comportamientos del consumidor adictivo miope y racional con variables de precios relativos, ingreso y dos indicadores para identificar modificaciones en el comportamiento de los consumidores ante la Covid-19 y la restricción de la movilidad de las personas.

El documento se conforma además de esta introducción por un apartado con las teorías económicas del comportamiento adictivo y la literatura relevante; la metodología con la especificación del modelo de cointegración para el consumo adictivo de cigarros, estimado con el método fmols para incorporar problemas de endogeneidad y la prueba de cambio estructural; los datos para la medición del consumo de cigarros y sus determinantes, y el análisis de los resultados de los modelos estimados como las pruebas estadísticas; $y$, al final se presentan una discusión con las implicaciones analíticas de los resultados y las conclusiones del estudio.

\section{Teoría económica de las adicciones y literatura relevante}

El comportamiento de los consumidores desde el punto de vista de la teoría de la elección tradicional implica que los individuos toman decisiones sobre los bienes que demandan en el periodo corriente considerando el precio de todos los productos que conforman su canasta y su restricción presupuestal o ingreso disponible. En tal sentido, los individuos que consumen cigarros son sensibles a los cambios de los precios como cualquier otro bien (Mas-Colell, Whinston, \& Green, 1995). Becker y Murphy (1988) construyeron la "Teoría de Adicción Racional" para modelar conductas maximizadoras de utilidad. Esto ayuda a entender el comportamiento de los individuos ante el posible consumo de algún bien adictivo y los posibles factores explicativos de estos comportamientos.

Cuando los consumidores cuentan con un plan consistente para maximizar su utilidad en el tiempo se plantea que son "racionales". De esta manera, los consumidores pueden tratar de anticipar las consecuencias futuras con relación a sus elecciones presentes. En esta teoría hasta las adicciones más fuertes son racionales en el sentido de que incorporan la idea de maximización de utilidad en el futuro tomando en cuenta las preferencias presentes. La ecuación (1) muestra que el consumo de un bien (cualquiera que sea) se encuentra en función del stock de consumo inicial de ese bien, de los precios del bien y de la utilidad marginal que le reporte su uso. 


$$
\int_{0}^{T} U[c(t), S(t), y(t)] e^{-\rho t}
$$

En donde $U(t)$ es la utilidad en el periodo $t, c(t)$ es el consumo adictivo del bien en el periodo $t$ y $S(t)$ es el stock de capital adictivo que depende del consumo pasado de $c$ y de los eventos del ciclo de vida, $y$ es el ingreso y $\rho$ es la tasa de descuento temporal. La principal condición en la teoría de "Adicción racional" para la existencia de comportamientos adictivos es la aparición de "estados estacionarios inestables" y "complementariedades adyacentes" que podrían llevar a las adicciones tanto "benéficas" como "perjudiciales".

La idea de "estados estacionarios inestables" se puede ver en la figura 1. La línea $s^{1} s^{1}$ representa un "estado estacionario estable" en $\delta s^{* 1}=c^{* 1}$, las flechas indican que cualquier desviación de $s^{* 1}$ causarán un regreso a este punto y a lo largo de la senda representada por $s^{1} s^{1}$. Mientras que la línea $s^{0} s^{0}$ muestra un equilibrio de "estado estacionario inestable" en $\delta s^{* 0}=c^{* 0}$, las flechas indican ahora que cualquier desviación de $s^{* 0}$ ocasionarán a su vez mayor desviación de manera que se provoca un comportamiento divergente alejándose del equilibrio sobre la senda $s^{0} s^{0}$.

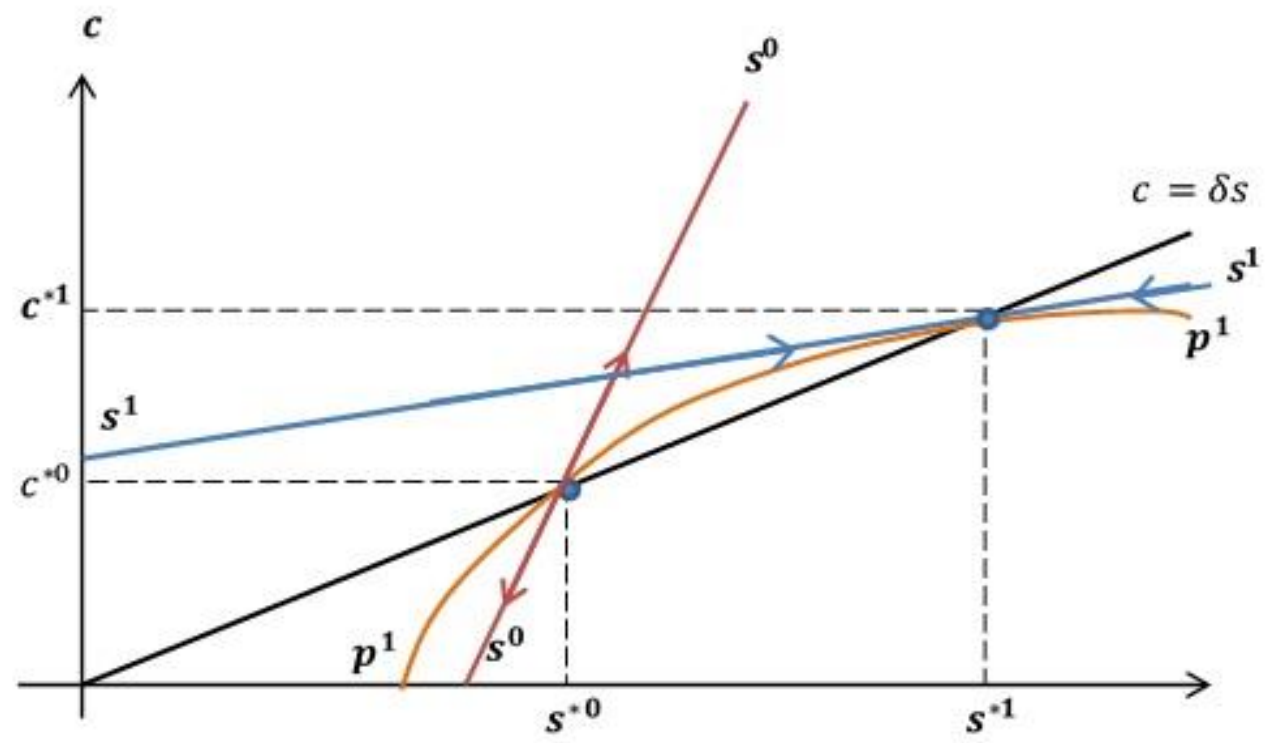

Figura 1. Estados estacionarios estables e inestables Fuente: Elaborado con base en Becker y Murphy (1988)

En el caso de que el incremento en el tiempo de la utilidad marginal que reporta un bien excede al incremento en el precio, entonces existirá "complementariedad adyacente", si la desigualdad se mantiene, significa que un incremento en el consumo pasado incrementa el consumo presente. La definición más elemental de "Adicción" es que una persona es potencialmente adicta a un bien siempre que un incremento en su consumo actual aumente su consumo futuro. Esto solamente ocurre si y sólo si su comportamiento muestra complementariedades adyacentes. Implica que un individuo es adicto a un bien sólo cuando el consumo pasado de ese bien incrementa la utilidad marginal del 
consumo presente. Sin embargo, tal efecto sobre la utilidad marginal es necesario, pero no suficiente para la adicción ya que ésta depende también de otros factores.

La relación entre adicción y complementariedad adyacente puede llevar a la conclusión de que un incremento anticipado en el precio futuro de los bienes adictivos reduce el consumo presente. Estos efectos negativos de los cambios anticipados en el precio futuro sobre el consumo presente es la manera más adecuada de distinguir la adicción racional o la formación de un hábito de un comportamiento miope. Si el grado de adicción es alto o la complementariedad entre el consumo pasado y el consumo presente es fuerte, el consumo de estado estacionario será inestable, el consumo se incrementará en el tiempo cuando se encuentra en niveles por arriba del estado estacionario y cae en el tiempo cuando se encuentra por debajo. Esta idea también muestra que aquellos individuos que descuentan el tiempo más rápido son más vulnerables a convertirse en adictos. Sin embargo, no se debe olvidar que existen otros factores económicos como el ingreso, los precios, la pérdida de empleo (Ben Lakhdar \& Bastianic, 2011) o la pérdida de algún ser querido, un divorcio, o cualquier otro fenómeno estresante que pueden desencadenar comportamientos adictivos (NCI, 2017).

Con la "Teoría de Adicción racional" es consistente inferir que la única manera de terminar con las adicciones más fuertes como el cigarro o el alcohol es mediante la "abstinencia súbita", que es el desuso abrupto del bien o sustancia (Becker \& Murphy, 1988). La evidencia empírica sobre la Teoría de Adicción Racional y sus implicaciones fue probada por los mismos Becker, Grossman y Murphy (1994) sobre la demanda de cigarrillos mediante un modelo de sección cruzada para Estados Unidos entre 1955 y 1985 encontraron elasticidades precio de largo plazo entre - 0.7 y 0.8 destacando que el consumo en cualquier año es menor siempre que los precios pasados y futuros se incrementen. En un estudio relativamente reciente Baltagi (2007) aplica la teoría de adicción racional y confirma el efecto adictivo al cigarro al encontrar elasticidades precio entre -0.42 y -0.69 en el largo plazo y en un rango de -0.56 a -2.04 para Estados Unidos. Grossman y Chaloupka (1998), utilizan un modelo de panel construido a partir de la información recolectada por la Universidad de Michigan sobre el uso de alcohol, cigarrillos y drogas ilegales para estimar la función de demanda por cocaína entre 1976 y 1985. Ellos encontraron que la elasticidad precio de la demanda de largo plazo fue de -1.6 mientras que, en el corto plazo, el comportamiento miope la llevó a su condición de inelasticidad en -0.95 probando el modelo de adicción racional.

Tomando en cuenta algunos factores de riesgo, la hipótesis de adicción racional se cumple reduciendo el consumo de bienes adictivos cuando se condiciona al grado de adicción considerando su sobrevivencia en el futuro (Clarke, 2000). Ferguson (2000) identificó el modelo de adicción racional aplicado al consumo de café concluyendo que la teoría funciona para identificar estados estacionarios estables y elasticidades mayores que la unidad para este bien. En un estudio que combina series de tiempo de 1964 a 1994 para trece países europeos sobre consumo de cigarros, Escario y Molina (2020) utilizaron variables sobre precio y consumo per cápita de manera rezagada para identificar la importancia del comportamiento pasado, futuro y variables instrumentales relacionadas con los programas preventivos de cáncer de pulmón. Con sus resultados encontraron que el efecto de los precios en el consumo de cigarros es diverso pero prueban la teoría de adicción racional en el sentido de la existencia de la utilidad intertemporal con comportamientos miopes asociados con la tolerancia y el reforzamiento del consumo.

Iwasaki et al. (2006) sostienen la importancia del modelo de adicción racional en el largo plazo cuando el precio observa un incremento derivado de políticas gubernamentales impositivas 
para tratar de desalentar el consumo de cigarrillos. Mediante datos anuales entre 1955 y 2002 de la industria del tabaco y variables de precios, consumo y advertencias sobre los riesgos, encontraron una elasticidad precio de la demanda de largo plazo de -1.61 y un efecto directo del ingreso disponible de los consumidores en el contexto de la teoría de la adicción racional. Liu et al. (2015), utilizan datos anuales sobre las ventas de cigarrillos en China entre 2005 y 2010 para probar la hipótesis de adicción racional haciendo diferencia en la sustitución entre marcas de cigarrillos. Considerando los incrementos en los precios derivados de la política impositiva del gobierno chino, encontraron que la elasticidad precio de la demanda de cigarros en el país asiático fue de -0.488 verificando la importancia de la política pública para atenuar el problema.

En el caso específico de los países latinoamericanos, Guindon (2018), después de elaborar una búsqueda bibliográfica de estudios relacionados, encontró que las elasticidades precio de corto plazo de la demanda de cigarrillos muestra comportamientos heterogéneos. Para Panamá, Guatemala y Colombia esta elasticidad de corto plazo se localizó entre -0.6 y -0.8 mientras en el largo plazo, la subida de precios tendría una reducción de hasta -1.0. En un estudio para Uruguay y Brasil estuvo entre -0.2 y -0.6 en el corto plazo y entre -0.4 y -1.4 en el largo plazo. Otro grupo conformado por Brasil, Chile y México mostró un comportamiento más extremo en el que las elasticidades de corto plazo se presentan entre -0.10 y -0.30 ajustando hasta -0.45 en el largo plazo. Para México, Olivera et al. (2010) utilizaron datos de la Encuesta Industrial Mensual (EIM), el Índice Nacional de Precios al Consumidor (INPC) y del PIB obtenidos del Instituto Nacional de Estadística y Geografía (INEGI) para construir un modelo de cointegración con rezagos temporales para medir el efecto de los precios y el ingreso en el consumo de cigarrillos entre 1994 y 2005. Los resultados arrojaron que la elasticidad precio de largo plazo fue -0.25 indicando que cualquier aumento en los precios reduciría, pero menos que proporcionalmente la demanda de cigarrillos.

En 2018, el Centro de Estudios de las Finanzas Públicas (CEFP) perteneciente a la cámara de diputados, en su nota informativa: "El tabaquismo y su efecto en las Finanzas Públicas: 2007 - 2017" identifica al consumo de tabaco como uno de los padecimientos prevenibles más costosos para el erario público (CEFP, 2018) y consigna que a pesar de que el Impuesto Especial sobre Productos y Servicios (IEPS) ha buscado elevar el precio de los cigarrillos y con ello restringir su consumo, en realidad no lo ha logrado. La principal razón para la situación anterior es que la elasticidad precio de la demanda de tabaco es demasiado pequeña pero no menciona la magnitud. Jiménez et al. (2008) retomaron los datos contenidos en la Encuesta Nacional de Ingreso y Gasto de los Hogares (ENIGH) para elaborar un modelo de datos panel para la demanda de cigarrillos que estima el efecto del precio en el consumo. Los resultados muestran que el precio es un gran determinante para las decisiones de los hogares sobre fumar y sobre la cantidad diaria de cigarrillos que se fuman; la elasticidad precio que estimaron fue -1.24 . 


\section{Metodología}

En la especificación del modelo para el consumo adictivo de cigarros para México se utiliza el planteamiento de Becker y Murphy (1988) y Becker, Grossman y Murphy (1991) con los componentes de comportamientos miope y racional, los precios relativos, el ingreso, así como los indicadores de la Covid-19 y restricción a la movilidad de las personas. Para eliminar los posibles sesgos de estimación por problemas de endogeneidad en las relaciones de cointegración se utiliza el método de estimación fmols y para el análisis de cambio estructural en modelos de cointegración se aplica el estadístico de la prueba de hipótesis $H_{s m}$ propuesto por Wagner y Wied (2015).

\subsection{Especificación del modelo de consumo adictivo de cigarros}

En la estructura básica del modelo de consumo adictivo de Becker y Murphy (1988) se considera que el consumo de cigarros actual $\left(C_{t}\right)$ se determina por el consumo inmediato anterior $\left(C_{t-1}\right)$ y el consumo esperado para el siguiente periodo $\left(C_{t+1}\right)$, así como de los precios relativos de los cigarros $\left(P r_{t}\right)$, el ingreso de los consumidores $\left(Y_{t}\right)$, la variable cualitativa con valores igual a uno desde marzo hasta diciembre de $2020\left(\operatorname{Cov} 19_{t}\right)$ y la movilidad de las personas $\left(\operatorname{ResMov}_{t}\right)$ de acuerdo a los mapas informes de tendencias de Apple (Apple, 2021); ver la ecuación 2.

$$
C_{t}=\alpha_{1} C_{t-1}+\alpha_{2} C_{t+1}+\beta_{1} \operatorname{Pr}_{t}+\beta_{2} Y_{t}+\beta_{3} \operatorname{Cov} 19_{t}+\beta_{4} \operatorname{ResMov}_{t}+u_{t}
$$

Para analizar los supuestos de consumo adictivo de cigarros miope y/o racional, la ecuación (2) se resuelve para encontrar el consumo de cigarros de equilibrio dado el consumo anterior (backward looking) y lo que se planea consumir (forward-looking) en el siguiente periodo (ecuación 3).

$$
C_{t}=\frac{\beta_{1} P_{t}+\beta_{2} Y_{t}+\beta_{3} \operatorname{Cov} 19_{t}+\beta_{4} \operatorname{ResMov}_{t}+u_{t}}{\left(1-\alpha_{1}-\alpha_{2}\right)}
$$

Las dos principales condiciones para definir el consumo adictivo de cigarros se obtienen al considerar que los parámetros $\alpha_{1}$ y $\alpha_{2}$ son diferente de cero. El consumo adictivo miope predomina si $\alpha_{1}>\alpha_{2}$ o en el caso extremo si $\alpha_{1}>0$ y $\alpha_{2}=0$. En tanto el consumo adictivo racional existe si se cumplen al mismo tiempo $\alpha_{1}>0$ y $\alpha_{2}>0$. Aunque en la literatura no está claro que predomina, el consumo rezagado o el planeado, en el enfoque del consumidor racional lo lógico es suponer que el consumo esperado debe predominar, por lo que se impone la condición de que $\alpha_{1}<\alpha_{2}$. Para que el equilibrio exista y sea estable es necesario que $\alpha_{1}+\alpha_{2}<1$.

De las hipótesis económicas adicionales sobre el consumo adictivo se encuentra la de la tasa de descuento individual $\rho$ del modelo de optimización inter-temporal de Becker y Murphy (1988), que se obtiene de la condición $\beta_{1}=1 /(1+\rho)$ donde $\alpha_{1}=\beta_{1} \alpha_{2}$ (Laporte, Rohi, \& Ferguson, 2017). Con el modelo de adicción racional se espera que la elasticidad precio relativo de los cigarros de largo plazo sea negativa y mayor a la de corto plazo. Aunque el consumo de cigarros sea inelástico al precio relativo en el corto plazo $\left(\left|\beta_{1}\right|<1\right)$, las condiciones sobre el consumo adictivo miope o racional 
garantizan que la elasticidad precio de largo plazo sea mayor a la de corto plazo; esto es, $\beta_{1} /\left(1-\alpha_{1}-\right.$ $\left.\alpha_{2}\right)>\beta_{1}$.

Tanto en el enfoque tradicional de la teoría económica convencional sobre la elección de los consumidores como en el planteamiento de Becker y Murphy (1988), el consumo de cigarros reacciona principalmente a los cambios de los precios relativos, lo cual implica que las elasticidades de los otros factores explicativos, como el ingreso, deben ser menores en el corto y en el largo plazo de los precios de cigarros; $\left|\beta_{1}\right|>$ a todas $\beta_{i}$ diferentes en la ecuación 3.

En la ecuación (2) para el consumo adictivo de cigarros se incluyeron adicionalmente dos indicadores con el objetivo de identificar los efectos de la Covid-19 en el patrón de consumo. La primera es una variable cualitativa $\left(\operatorname{Cov} 19_{t}\right)$ con valores igual a uno desde marzo hasta diciembre de 2020 , donde su parámetro $\left(\beta_{3}\right)$ mide el efecto de la pandemia sobre el consumo de cigarros. En el caso de que el parámetro sea positivo indica que, debido a la pandemia, el consumo de cigarros aumentó y si es negativo el consumo de cigarros se redujo.

El segundo indicador $\left(\operatorname{ResMov}_{t}\right)$ mide la restricción a la movilidad de las personas con el promedio de la proporción de solicitudes de indicaciones de las personas en Mapas informes de tendencias de movilidad de las personas de Apple (Apple, 2021) del mes con respecto a las solicitudes promedio que se tenían en el mes de febrero de 2020 para tres tipos de medios de movilidad: caminando, manejando o en el trasporte público.

El indicador de ( $\left.\operatorname{ResMov}_{t}\right)$, en tanto por uno, muestra tres posibilidades: 1) Cuando el valor del indicador es uno muestra que la movilidad de las personas es la misma al mes de referencia por lo que no existe restricción; 2) En el caso donde el valor del indicador es menor a uno la movilidad de las personas se reduce con respecto al mes de referencia por lo que se concluye que existe una restricción; y, 3) La última posibilidad es que el valor del indicador sea mayor a uno que implica que la movilidad aumentó con respecto a la que se tenía en el mes de referencia.

De acuerdo con lo anterior, el planteamiento es que la menor movilidad de las personas ( $\operatorname{ResMov}_{t}<1$ ) tiene como consecuencia el menor consumo de cigarros durante el periodo de la pandemia si el signo del parámetro $\beta_{4}$ es positivo y se provocará un aumento cuando el parámetro sea negativo.

\subsection{Método de estimación fmols y estadístico $H_{s m}$ para prueba de hipótesis de cambio estructural}

En los modelos de la demanda de consumo de bienes en mercados microeconómicos o desagregados, en particular para el caso del consumo de cigarros, es común encontrar problemas de endogeneidad que afecta a los precios relativos debido a su doble causalidad con el consumo de cigarros demandado y por otros factores que pueden provocar un sesgo de estimación de los parámetros. El método de estimación más utilizado para eliminar el sesgo de endogeneidad en modelos cointegrados es el Fully Modified OLS (fmols) propuesto por Phillips y Hansen (1990), que tiene la ventaja de ser compatible con las pruebas de cambio estructural de Wagner y Wied (2015).

Para desarrollar el método de estimación fmols establecemos que $X_{t}^{\prime}$ es el vector de variables explicativas incluyendo las vinculadas al consumo de cigarros miope y racional; esto es, $X_{t}^{\prime}=\left[C_{t-1}\right.$, 
$\left.C_{t+1}, P r_{t}, Y_{t}, \operatorname{Cov} 19_{t}, \operatorname{ResMov}_{t}\right]$. De acuerdo con (Vogelsang \& Wagner, 2014; Wagner \& Wied, 2015) la relación de cointegración entre $C_{t}$ y $X_{t}^{\prime}$ se puede escribir con el siguiente sistema de ecuaciones:

$$
\begin{gathered}
C_{t}=D^{\prime}{ }_{t} \theta_{D}+X^{\prime}{ }_{t} \theta_{X}+u_{t} \\
X_{t}=X_{t-1}+v_{t}
\end{gathered}
$$

En donde $D_{t}^{\prime} \in \mathbb{R}$ se identifica como la tendencia determinística que pertenece al conjunto doble de los reales; el vector de innovaciones conjuntas $\eta_{t}=\left(u_{t}, v_{t}^{\prime}\right)^{\prime}$ cumple con subindices de tiempo que pertenecen al conjunto doble de los enteros $\left(\eta_{t}\right)_{t \in \mathbb{Z}}$, sigue un proceso estacionario descrito por $\frac{1}{\sqrt{T}} \sum_{t=1}^{[s T]} \eta_{t} \Rightarrow \Omega^{1 / 2} W(s)$, donde $W(s)$ indica un movimiento Browniano estándar, $s$ es una proporción con valores $(0 \leq s \leq 1)$ y $\Omega<\infty$ es la varianza de largo plazo de $\left(\eta_{t}\right)_{t \in \mathbb{Z}}$ con

$$
\Omega=\left[\begin{array}{ll}
\Omega_{u u} & \Omega_{u v} \\
\Omega_{v u} & \Omega_{v v}
\end{array}\right]:=\sum_{j=-\infty}^{\infty} \mathbb{E}\left(\eta_{t} \eta_{t-j}^{\prime}\right)
$$

Para el desarrollo del método de estimación Wagner y Wied (2015) mencionan que es importante usar:

$$
\Omega^{1 / 2}=\left[\begin{array}{cc}
\omega_{u \cdot v} & \lambda_{u v} \\
0 & \Omega_{v v}^{1 / 2}
\end{array}\right]
$$

donde $\omega_{u \cdot v}^{2}=\Omega_{u u}-\Omega_{u v} \Omega_{v v}^{-1} \Omega_{v u} \mathrm{y} \lambda_{u v}:=\Omega_{u v}\left(\Omega_{v v}^{1 / 2}\right)^{-1}$

El supuesto de $\Omega_{v v}>0$ impone una restrición para la existencia de cointegración, por lo que representa una de las razones para usar el método de estimación fmols. En el caso de que $C_{t} \sim I(1) \mathrm{y}$ $X_{t} \sim I(1)$ estén cointegrados, pero las variables $X_{t}$ no sean estrictamente exógenas y no se puedan sostener los supuestos de endogeneidad débil (Spanos, 2003; Afonso, 2013; Vogelsang \& Wagner, 2014), el estimador fmols elimina los sesgos de endogeneidad con el siguiente procedimiento. El estimador fmols emplea los resultados de la estimación de las ecuaciones (4) a la (7), para obtener las innovaciones estimadas $\hat{\eta}_{t}=\left(\hat{u}_{t}, \hat{v}_{t}^{\prime}\right)^{\prime}$, transformar la variable endógena como $C_{t}^{+}:=C_{t}-$ $\Delta X_{t}^{\prime} \widehat{\Omega}_{v v}^{-1} \widehat{\Omega}_{v u}$ y con ello construir el estimador como en la ecuación (8):

$$
\begin{gathered}
\hat{u}_{t, m}^{+}:=C_{t}^{+}-D^{\prime}{ }_{t} \hat{\theta}_{D, m}-X^{\prime}{ }_{t} \hat{\theta}_{X, m} \\
=C_{t}-\Delta X^{\prime}{ }_{t} \widehat{\Omega}_{v v}^{-1} \widehat{\Omega}_{v u}-D^{\prime}{ }_{t} \hat{\theta}_{D, m}-X^{\prime}{ }_{t} \hat{\theta}_{X, m} \\
=u_{t}-v^{\prime}{ }_{t} \widehat{\Omega}_{v v}^{-1} \widehat{\Omega}_{v u}-D^{\prime}{ }_{t}\left(\hat{\theta}_{D, m}-\theta_{D}\right)-X^{\prime}{ }_{t}\left(\hat{\theta}_{X, m}-\theta_{X}\right)
\end{gathered}
$$

donde $\hat{\theta}_{D, m}$ y $\hat{\theta}_{X, m}$ los parámetros estimados con el método $f m l s, \hat{\Omega}\left(=\hat{\Omega}_{m T}\right)$ es la varianza de largo plazo estimada, todos ellos computados con muestras de $1, \ldots,[m T]$.

Wagner y Wied (2015) proponen el estadístico $H_{s m}$ para la prueba de hipótesis de cambio estructural en los parámetros de las relaciones de cointegración, sin sesgo de endogeneidad y 
estimados con el método fmols. El estadístico $H_{s m}$ identifica cambios en las relaciones de cointegración de acuerdo al proceso de suma acumulada parcial de los residuales modificados con el método de estimación fmols y se define como en la siguiente ecuación (9):

$$
H_{s m}=\frac{1}{\widehat{\omega}_{u \cdot v}^{2}}\left(\frac{1}{T} \sum_{i=[m T]+1}^{[s T]}\left(\frac{1}{\sqrt{T}} \hat{S}_{i}^{+}\right)^{2}-\frac{1}{T} \sum_{i=1}^{[m T]}\left(\frac{1}{\sqrt{T}} \hat{S}_{i}^{+}\right)^{2}\right)
$$

donde el factor de escala es un estimador consistente de la varianza condicionada de largo plazo $\widehat{\omega}_{u \cdot v}^{2}=\hat{\Omega}_{u u}-\hat{\Omega}_{u v} \hat{\Omega}_{v v}^{-1} \hat{\Omega}_{v u}$.

\section{Datos y Resultados}

\subsection{Datos}

El consumo de cigarros se construyó con las ventas de cajetillas de cigarros mensuales de la Encuesta Mensual de la Industria Manufacturera (EMIM) del INEGI, para el periodo de enero de 2005 a diciembre de 2020.

Para la medición de la población fumadora mensual se utilizaron la Encuesta Nacional de Adicciones 2002 (ENA-2002) elaborada por el INEGI; la Encuesta Nacional de Adicciones 2011 (ENA2011) elaborada de manera conjunta por el Instituto Nacional de Salud Pública (INSP) y el Instituto Nacional de Psiquiatría "Ramón de la Fuente Muñiz" (INPRFM); y, la Encuesta Nacional de Salud y Nutrición (ENSANUT) de 2018 elaborada por el INEGI y el INSP.

Con los datos anuales de cada encuesta se aplicó el método de interpolación simple para obtener una medición mensual de la población fumadora. El método de interpolación supone que la población fumadora creció linealmente entre los dos periodos, tal que comparar los datos de población entre los extremos se puede dividir entre $n$ cantidad de meses $\left.\left(\left(\mathrm{P}_{2018} / \mathrm{P}_{2011}\right)\right)-1\right) / n$. Es importante mencionar que para la estimación de la población fumadora de 2018 a 2021 suponemos que se mantuvo la tendencia negativa y que no se provocó un aumento significativo de personas fumadoras por la pandemia. El consumo de cigarros por persona fumadora es la razón de las ventas de cajetillas de cigarros a personas fumadoras por mes.

El método X-13 ARIMA-SEATS se aplicó para desestacionalizar el consumo de cigarros por persona fumadora mensual. Para la medición de los precios relativos de los cigarros se utiliza el índice de precios al consumidor para cigarrillos entre el índice de precios al consumidor total mensual de 2005 a 2020 del INEGI. El Indicador Global de Actividades Económicas (IGAE) desestacionalizado del INEGI se considera como indicador del comportamiento del ingreso mensual. Como se menciona en el apartado 3.1 para la medición de la restricción a la movilidad de las personas se utiliza el promedio de la proporción de solicitudes de indicaciones de las personas en Mapas informes de tendencias de movilidad de las personas de Apple (Apple, 2021) del mes con respecto a las solicitudes promedio que se tenían en el mes de febrero de 2020 para tres tipos de medios de movilidad: caminando, manejando o en el trasporte público. 


\subsection{Tendencias del consumo de cigarros y sus determinantes}

Con los resultados de la Encuesta Nacional de Salud y Nutrición (ENSANUT) de 2018 se confirma un cambio de tendencia en la población fumadora al pasar de 16.4 a 17.3 millones de personas de 2002 a 2011 y a 16.1 millones de personas en 2018. La prevalencia a fumar es de $17.9 \%$ en 14.8 millones de adultos y $5.7 \%$ en 1.3 millones de adolescentes que suman los 16.1 millones de personas fumadoras en 2018 (INEGI-INSP, 2018). Con tal tendencia y de acuerdo con nuestras estimaciones, la población fumadora sería de 15.9 millones de personas al momento de la pandemia en 2020; 250 mil personas menos a las observadas por la ENSANUT en 2018; ver cuadro 1.

Aunque la prevalencia de la población fumadora se redujo, al mismo tiempo el consumo mensual de cajetillas por persona fumadora aumentó al pasar de 7.5 en 2011 a 14.2 en 2018, lo cual significa que el consumo adictivo de cigarros aumentó 89\% en tan sólo siete años. En específico, el crecimiento acelerado del consumo de cigarros se observa a partir de 2015; ver cuadro 1 y primera gráfica de la figura 2 .

Es importante hacer notar que la tendencia en el consumo de cigarros fue diferente en los periodos previos a la pandemia, donde de forma consistente se observaron reducciones de $-4.32 \%$ de 2005 a 2010 y de $-10.14 \%$ en el periodo de 2011 a 2014; ver consumo de cajetillas al mes por persona desestacionalizado en el cuadro 1 y primera gráfica de la figura 2.

Con la crisis sanitaria de la Covid-19 se observa la reducción del consumo de cigarros hasta -25\% en promedio de marzo a diciembre de 2020 (ver cuadro 1). La caída del consumo más profunda se observa en el segundo trimestre (abril-junio) y, posterior a ello, se tiende hacia la recuperación de los niveles del consumo de cigarros pre-pandemia; ver primera gráfica de la figura 2. Cabe mencionar que este segundo trimestre de 2020 fue el que mayor confinamiento representó, se cerraron escuelas y oficinas, los consumidores tuvieron que refugiarse en sus hogares, dejar la convivencia con amigos y muchos de ellos perdieron sus empleos o les redujeron sus sueldos por lo que la combinación de estos factores pudiera explicar la reducción en el consumo de cigarrillos en este periodo.

De acuerdo con la Política Pública uno de los mecanismos para reducir el consumo de cigarros es por medio de la instrumentación de nuevos impuestos o el aumento de los ya existentes. Desde el punto de vista del análisis estadístico, la aplicación del aumento de los impuestos se manifiesta en el comportamiento de los precios relativos, donde se compara la diferencia entre el aumento de los precios de los cigarros con respecto a todos los bienes y servicios medidos con el índice de precios al consumidor.

Con los estadísticos del cuadro 1 se identifica que las mayores reducciones en el consumo de cigarros se vinculan con el crecimiento de los precios relativos. La tasa de crecimiento de los precios de los cigarros aumentó seis puntos por arriba de la inflación en promedio en los periodos 20052010 y 2011-2014. Con la gráfica 2 de la figura 2 sobre el crecimiento de los precios relativos se identifican claramente el efecto de los impuestos aplicados por el gobierno federal donde lo más importante son los saltos observados en los primeros meses de 2011.

Por otro lado, el consumo de los cigarros tiende aumentar cuando el crecimiento del precio de los cigarros no es suficientemente mayor a la inflación y esto se observa claramente en el periodo prepandemia de 2015 a 2019. El precio de los cigarros aumentó $0.84 \%$ por arriba de la inflación en este periodo; ver cuadro 1 y gráfica de precios relativos en la figura 2. 
En los primeros meses de 2020, incluyendo marzo, junto con la instrumentación de las medidas para contener la transmisión de la pandemia por el Covid-19 se aplicaron incrementos en los impuestos que provocaron que los precios de los cigarros aumentaran $9.6 \%$ por arriba de la inflación en todo el año.

Cuadro 1. Consumo de cigarros y sus determinantes económicos en México, 2005-2020

\begin{tabular}{|l|c|c|c|c|c|}
\hline \multicolumn{1}{|c|}{ Variables } & $\begin{array}{c}\mathbf{2 0 0 5} \mathbf{a} \\
\mathbf{2 0 1 0}\end{array}$ & $\begin{array}{c}\mathbf{2 0 1 1} \mathbf{\mathbf { a }} \\
\mathbf{2 0 1 4}\end{array}$ & $\begin{array}{c}\mathbf{2 0 1 5} \mathbf{2 0 1 9} \\
\mathbf{2 0 1}\end{array}$ & $\begin{array}{c}\text { Enero a } \\
\text { diciembre de } \\
\mathbf{2 0 2 0}\end{array}$ & $\begin{array}{c}\text { Marzo a } \\
\text { diciembre de } \\
\mathbf{2 0 2 0}\end{array}$ \\
\hline $\begin{array}{l}\text { Población fumadora (ENA, 2002 y } \\
\text { 2011; ENSANUT, 2018) }\end{array}$ & 16.37 & 17.29 & 16.12 & & \\
\hline $\begin{array}{l}\text { Población fumadora estimada } \\
\text { (millones de personas) }\end{array}$ & 16.87 & 17.10 & 16.42 & 15.90 & 15.89 \\
\hline $\begin{array}{l}\text { Cajetillas al mes por persona } \\
\text { fumadora }\end{array}$ & 11.22 & 8.30 & 13.19 & 11.61 & 9.67 \\
\hline tasa de crecimiento & -3.49 & -9.89 & 12.03 & -21.21 & -22.56 \\
\hline $\begin{array}{l}\text { Cajetillas al mes por persona } \\
\text { fumadora (desestacionalizado) }\end{array}$ & 14.94 & 8.48 & 12.95 & 12.75 & 12.62 \\
\hline tasa de crecimiento & -4.48 & -10.07 & 12.84 & -17.92 & -18.88 \\
\hline $\begin{array}{l}\text { Tasa de crecimiento de los precios } \\
\text { relativos de las cajetillas }\end{array}$ & 5.13 & 6.64 & 0.84 & 9.62 & 9.25 \\
\hline $\begin{array}{l}\text { Tasa de crecimiento de los precios } \\
\text { de las cajetillas }\end{array}$ & 9.47 & 10.40 & 4.78 & 12.96 & 12.58 \\
\hline $\begin{array}{l}\text { Tasa de crecimiento de los precios } \\
\text { al consumidor }\end{array}$ & 4.34 & 3.76 & 3.94 & 3.34 & 3.32 \\
\hline $\begin{array}{l}\text { Tasa de crecimiento del IGAE } \\
\text { (desestacionalizado) }\end{array}$ & 1.37 & 2.82 & 1.90 & -8.76 & -10.25 \\
\hline Movilidad: caminando & & & & -34.66 & -41.59 \\
\hline Movilidad: manejando & & & -66.18 & -79.32 \\
\hline Movilidad: transporte & & & -72 \\
\hline
\end{tabular}

Fuente: Elaborado con datos del INEGI y Mapas informes de tendencias de movilidad de las personas de Apple 

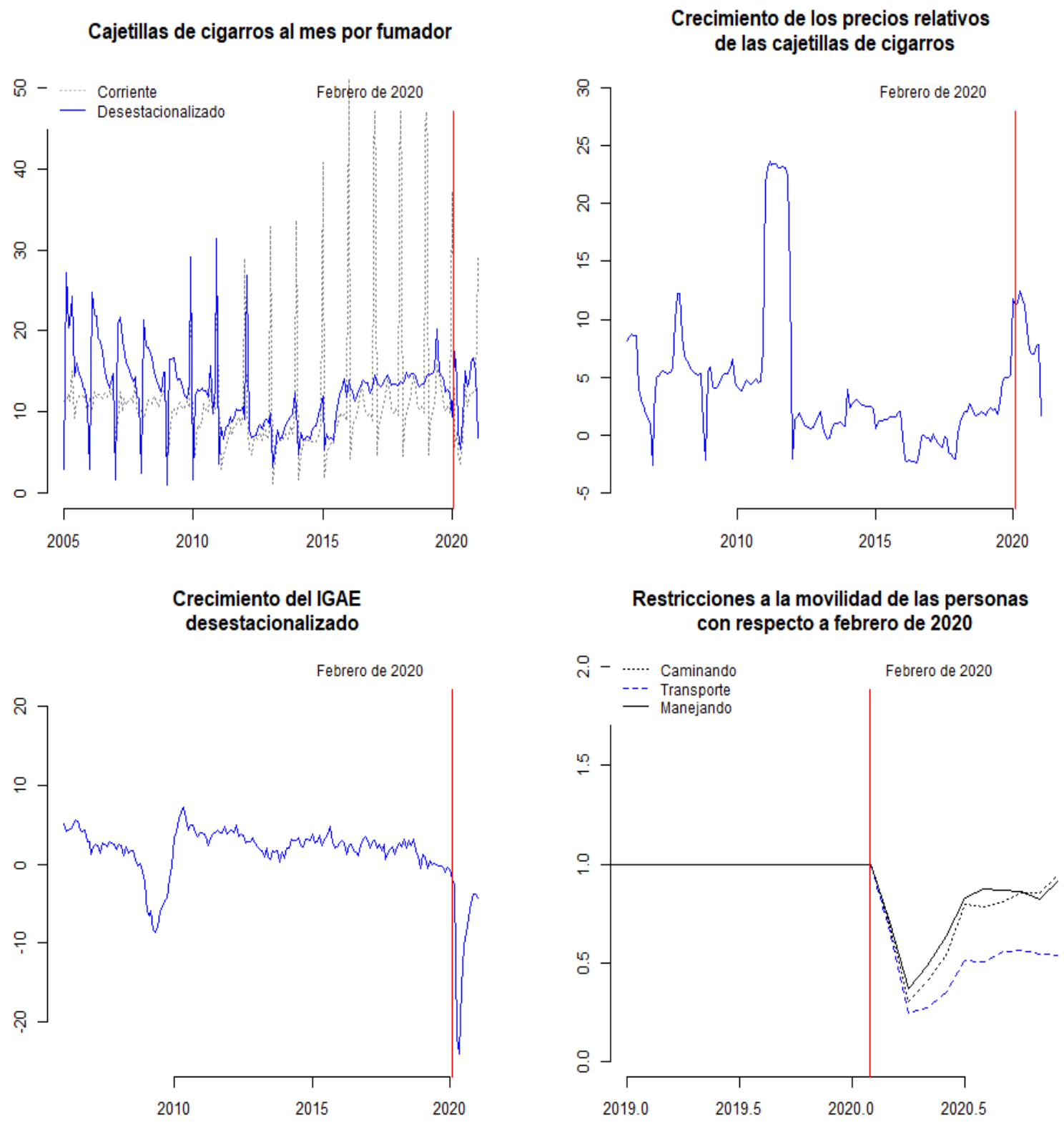

Figura 2. Consumo de cigarros y sus determinantes económicos en México, 2005-2020

El ingreso es el otro componente importante para explicar el comportamiento del consumo de cigarros. A pesar de la crisis de 2009 y el crecimiento moderado en 2013, las tasas de crecimiento promedio del ingreso (igae) de 1.4\% y 2.82\% no parecen ser la principal explicación de la reducción del consumo de cigarros en los periodos 2005-2010 y 2011-2014 respectivamente; ver cuadro 1. Por otro lado, el crecimiento moderado del ingreso de 1.9\% pudo ser una de las causas del aumento del consumo del cigarro en el periodo 2015-2019. Pero la reducción del ingreso en $-10.3 \%$ de marzo a diciembre sería otro de los factores que provocaron la contracción del consumo de cigarros en 2020; ver cuadro 1 y gráfica de crecimiento del igae en la figura 2.

Con el indicador sobre la restricción de la movilidad de las personas, que aplica solamente al 2020, se observa que durante el segundo trimestre del año se redujeron drásticamente los tres tipos 
de movilidad (caminando, manejando y transporte público). Después de ello, mientras que la restricción en la movilidad de las personas caminando y manejando se relajó, la vinculada al transporte público se mantuvo. De tal forma que la restricción a la movilidad en el transporte público de $79.4 \%$ se mantuvo prácticamente al doble de la restricción de $34.3 \%$ manejando y $41.6 \%$ caminando durante los meses de marzo a diciembre de 2020.

\subsection{Resultados}

Para entender el comportamiento de los consumidores de cigarros pre-pandemia y durante la pandemia se estimaron diferentes versiones del modelo de consumo adictivo miope y/o racional de la ecuación 2. Todas las variables se utilizaron en logaritmo natural por lo que los parámetros miden elasticidades.

En el modelo 1 se consideran los componentes del consumo rezagado y futuro $\left(C_{t-1}\right.$ y $\left.C_{t+1}\right)$, los precios relativos de los cigarros $\left(\mathrm{Pr}_{t}\right)$ y el ingreso $\left(\operatorname{Igae}_{t}\right)$ para el periodo de enero de $2005 \mathrm{a}$ febrero de 2020 para tener un panorama pre-pandemia sobre el comportamiento de los consumidores de cigarro en México. Los resultados muestran que los parámetros del consumo rezagado y futuro no son significativos, que el consumo de cigarros es elástico a los precios relativos (-1.6) pero es más elástico al ingreso (3.03).

En el modelo 2 se consideran las mismas variables, pero se estima con la muestra completa de datos para incluir el periodo de pandemia; esto es, de enero de 2005 hasta diciembre de 2020. Ahora los resultados indican que los componentes del consumo rezagado y futuro son significativos y positivos, los consumidores de cigarros son sensibles a las modificaciones de los precios relativos y son mucho más sensibles a los cambios de sus ingresos. A diferencia del modelo 1 de pre-pandemia, con el modelo 2 las elasticidades precios relativos y el ingreso de corto plazo, -1.15 y 2.13 respectivamente, son menores a las elasticidades de largo plazo (-1.6 y 3.0).

Los resultados del modelo 1 y 2 son relevantes debido a que previo a la pandemia los consumidores de cigarros no se comportan de acuerdo con la hipótesis de la teoría de las adicciones miope y racional. Sin embargo, cuando se considera el contexto de la crisis sanitaria y económica existe evidencia de un comportamiento intertemporal de los consumidores de acuerdo con la teoría de las adicciones miope y racional de Becker y Murphy (1988), donde los consumidores ajustan rápidamente a los cambios de los precios relativos y sobre todo del ingreso.

Con el tercer modelo se revisan las implicaciones analíticas al incorpora la variable cualitativa $\operatorname{Cov} 19_{t}$ que mide el efecto específico de la crisis sanitaria y económica sobre el consumo de cigarros. Los resultados muestran un parámetro significativo y positivo, lo cual indica que la crisis sanitaria y económica tuvo como efecto un aumento del consumo de cigarros tal como sostiene la teoría de adicción racional al referirse al impacto de eventos estresantes como la pérdida de empleo. También se encontró que el consumo rezagado y futuro dejan de ser relevantes, y se fortalece la importancia de los precios relativos y el ingreso en las decisiones de los consumidores de cigarros.

En el cuarto modelo se analiza de manera conjunta los efectos de la variable cualitativa $\operatorname{Cov} 19_{t}$ y la restricción a la movilidad de las personas $\operatorname{ResMov}_{t}$ con tres tipos de movilidad: Caminando, manejando y en el transporte público. Los resultados muestran que la variable 
cualitativa como los tres indicadores de restricción a la movilidad de las personas no son significativos. Pero se mantienen los resultados del tercer modelo con respecto el consumo rezagado y futuro, como la importancia de los precios relativos y el ingreso.

La implicación relevante de los resultados del modelo anterior es que posiblemente la variable cualitativa $\operatorname{Cov} 19_{t}$ y los tres tipos de restricción a la movilidad de las personas son dos maneras de medir el mismo efecto. En el modelo cinco se consideran los tres tipos de restricciones a la movilidad y los resultados muestran que la restricción a la movilidad en el transporte público es la variable significativa.

Cuadro 2. Modelos de consumo adictivo de cigarros en México, 2005-2020.

\begin{tabular}{|l|c|c|c|c|c|c|}
\hline Variables & Modelo 1 & Modelo 2 & Modelo 3 & Modelo 4 & Modelo 5 & Modelo 6 \\
\hline \multicolumn{1}{|c|}{$C_{t-1}$} & 0.079 & 0.167 & 0.075 & 0.075 & 0.079 & 0.081 \\
\hline p.value & 0.284 & 0.019 & 0.287 & 0.287 & 0.262 & 0.245 \\
\hline \multicolumn{1}{|c|}{$C_{t+1}$} & 0.108 & 0.174 & 0.102 & 0.104 & 0.103 & 0.101 \\
\hline p.value & 0.143 & 0.015 & 0.143 & 0.138 & 0.142 & 0.148 \\
\hline \multicolumn{1}{|c|}{ Pr $_{t}$} & -1.620 & -1.015 & -1.664 & -1.652 & -1.653 & -1.656 \\
\hline p.value & 0.000 & 0.000 & 0.000 & 0.000 & 0.000 & 0.000 \\
\hline \multicolumn{1}{|c|}{ Igae $_{t}$} & 2.974 & 1.913 & 2.995 & 2.968 & 2.981 & 2.987 \\
\hline p.value & 0.000 & 0.001 & 0.000 & 0.000 & 0.000 & 0.000 \\
\hline \multicolumn{1}{|c|}{ Cov19 $_{t}$} & & & 0.409 & 0.539 & & \\
\hline p.value & & & 0.004 & 0.395 & & \\
\hline Caminando $_{t}$ & & & & 0.676 & 1.453 & \\
\hline p.value & & & & 0.825 & 0.617 & \\
\hline${\text { Manejand } o_{t}}$ & & & & -0.649 & -0.938 & \\
\hline p.value & & & & 0.843 & 0.774 & \\
\hline Transport $_{t}$ & & & & 0.155 & -1.137 & -0.774 \\
\hline p.value & & & & 0.926 & 0.041 & 0.004 \\
\hline \multicolumn{1}{|c|}{$H_{\text {sm }}$} & 0.084 & 0.081 & 0.081 & 0.081 & 0.081 & 0.104 \\
\hline p.value & 0.100 & 0.100 & 0.100 & 0.100 & 0.100 & 0.100 \\
\hline
\end{tabular}

Nota: Para la estimación de los modelos de cointegración con el método fmols se utiliza distribuciones kernel, con bandwidth tipo and, la muestra $(\mathrm{m})$ se restringe a 0.9 para las pruebas de cambio estructural y para la estimación de la varianza con simulaciones de Monte Carlo. No se incluyeron los estadisticos de robustez, cmo la $\mathrm{R}^{2}$, debido a que las librerías de $\mathrm{R}$ (cointReg y cointmonitorR) utilizadas no las incluyen en sus resultados.

En el modelo seis se incluye sólo la restricción a la movilidad de las personas en el transporte público y los resultados son equivalentes a los encontrados con el modelo tres que considera la variable cualitativa $\operatorname{Cov} 19_{t}$. Dado que la restricción a la movilidad de las personas en el transporte público implica una reducción, al multiplicarse con el parámetro estimado negativo (-0.686) el efecto final es positivo. Por ello, la restricción de la movilidad de las personas en el transporte público tuvo un efecto positivo en el consumo de cigarros durante la crisis sanitaria y económica en 2020 pero no compensa el efecto negativo del aumento del precio y la reducción del ingreso, esto implica también 
que se vio limitada la interacción de los consumidores con amigos e incluso con familiares y con los entornos propicios para el consumo de tabaco.

El resultado anterior es importante ponerlo en el contexto donde los consumidores de cigarros no toman decisiones de forma intertemporal de acuerdo con la teoría de las adicciones miope y racional. Pero si ajustan sus decisiones de consumo de manera inmediata y son muy sensibles a los precios relativos y sobre todo a los cambios del ingreso.

Por último, los resultados del estadístico $H_{S m}$ muestra que en los seis modelos no existe evidencia de cambios estructurales de los parámetros por lo que las inferencias sobre los resultados de los modelos pre-pandemia y con crisis sanitaria y económica son robustas.

\section{Discusión y conclusiones}

En esta investigación nos enfocamos en analizar los efectos de la pandemia de la Covid-19 y la restricción a la movilidad de las personas sobre el consumo adictivo de cigarros en México en el periodo 2005-2020. Para ello se utiliza el marco analítico de la teoría de adicción racional de Becker y Murphy (1988), donde plantean que los consumidores adictivos consideran decisiones de acuerdo con una elección intertemporal que toma en cuenta el consumo inmediato pasado y futuro cercano, comportamiento miope y racional, y por ello son más sensibles a los cambios de los precios relativos de los cigarros.

En los primeros trabajos que se elaboraron para probar la hipótesis del consumo adictivo de cigarros a nivel internacional se prueba la existencia de comportamientos miope y racional donde el consumo pasado y futuro son importantes, inelásticos (menor a uno) a los precios de los cigarros en el corto plazo y elásticos, pero cercanos a la unidad en el largo plazo. En estudios actuales se ha encontrado que los consumidores adictivos a los cigarros, en el sentido de la teoría de la adicción racional de Becker y Murphy (1988), son cada vez más sensibles a los cambios de los precios relativos, pero también responden a los cambios del ingreso, entre otros. Ben Lakhdar y Bastianic (2011) muestran que ante una crisis económica se reduce el ingreso disponible de las personas y su poder adquisitivo, por lo que los individuos con comportamiento racional modifican su consumo para mantener el mismo nivel de satisfacción. En consecuencia, los fumadores actúan de forma racional ante un choque de ingreso al demandar menos cantidad de cigarros y, con ello, gastar menos dinero para obtener el mismo nivel de utilidad.

Para el caso de México se han estimado modelos sobre el consumo de cigarros considerando el contexto de la teoría económica convencional sobre la elección de los consumidores que sostiene que los fumadores son sensibles a los cambios de los precios como cualquier otro bien (Mas-Colell, Whinston, \& Green, 1995). Los resultados son contrastantes debido a que se muestra que los consumidores de cigarros no modifican su comportamiento con los cambios de los precios (CEFP, 2018), pueden modificar su consumo sólo si existen grandes cambios en los precios de los cigarros (Olivera, Cermeño, Sáenz de Miera, Jiménez, \& Reynales, 2010) o los consumidores son muy sensibles (elasticidad mayor a uno) a los precios relativos (Jiménez, Sáenz de Miera, Reynales, Water, \& Hernández, 2008). 
De acuerdo a nuestras estimaciones de los modelos de consumo adictivo de cigarros para México en el periodo 2005-2020, en el contexto de las teorías económicas sobre el consumo adictivo miope o racional y modelos de cointegración fmols, con endogeneidad y cambio estructural, los resultados encontrados indican que cambios no previstos en los precios de los cigarrillos proveen elasticidades precio de la demanda de cigarros estadísticamente significativos y mayores a la unidad (-1.15) tanto en el corto plazo como en el largo plazo (-1.6) comprobando que los consumidores hacen ajustes rápidos o inmediatos en sus niveles de consumo de cigarros. Algunos autores han encontrado elasticidades precio superiores a la unidad (Grossman \& Chaloupka, 1998; Iwasaki, Tremblay, \& Horton, 2006) y cercanas a -1.6. Estos resultados son consistentes con el planteamiento donde aumentos en el consumo actual o pasado genera que el consumo futuro también se incremente y que ese aumento sea aún mayor (como indica la "teoría de adicción racional"), solamente un incremento no previsto en el precio de los cigarrillos podría tener el efecto deseado por cualquier política pública que esté tratando de desincentivar el consumo de cigarros (Becker, Grossman, \& Murphy, 1991). Olivera et al. (2010) encontraron para México que la elasticidad precio de largo plazo fue -0.25 indicando que cualquier aumento en los precios reduciría, pero menos que proporcionalmente la demanda de cigarrillos. Jiménez et al. (2008) con datos de la ENIGH encontraron que el precio es un gran determinante para las decisiones de los hogares sobre fumar y sobre la cantidad diaria de cigarrillos que se fuman; la elasticidad precio que estimaron fue -1.24. Los estudios previos muestran resultados heterogéneos dependiendo de la fuente de los datos, la población objetivo, la magnitud del cambio en el precio y la metodología empleada para la estimación por lo que la elasticidad encontrada es consistente.

Adicionalmente, también se puede asegurar que el incremento que se ha observado en los últimos años en el precio de la cajetilla de cigarrillos a través de la política impositiva en México ha jugado un papel muy importante en la reducción del consumo, tal como argumentan Iwasaki et al. (2006) al mencionar el efecto directo sobre el ingreso disponible de los consumidores o el efecto sustitución hacia marcas más baratas de cigarrillos en China planteado por Liu et al. (2015). Pero, solamente cuando ese incremento es mayor a la tasa inflacionaria tendrá el efecto deseado de reducción del consumo. En tanto, la elasticidad ingreso encontrada se sitúa en promedio en 2.5, ello agrega una condición más a la construcción efectiva de la política impositiva pues el gravamen deberá ser suficientemente grande para compensar la tasa de inflación y el mayor poder adquisitivo de los consumidores por aumentos en los ingresos. Solamente de este modo el incremento de precios vía impuestos permitirá la reducción de la demanda de cigarros en México.

Dentro de algunos otros factores que determinan el consumo, o por lo menos el comienzo, de bienes adictivos como los cigarros, se encuentran aquellos eventos inesperados que generan gran cantidad de estrés y que por sus características se consideran como eventos detonantes de comportamientos de este tipo (Becker, Grossman, \& Murphy, 1991). Un ejemplo muy claro de ello es el confinamiento que gran parte de la población mundial y en particular en México ha sufrido desde hace más de un año y que ha cambiado el comportamiento en general de los individuos y en particular de los consumidores y, sobre todo, la manera en que se trabaja y en que se transporta la población. Los viajes en transporte público en México disminuyeron drásticamente eliminando prácticamente el 80\% de los mismos (Apple, 2021).

La mejor prueba de este otro tipo de determinantes planteados por la "Teoría de adicción racional" es el hallazgo sobre la importancia del efecto específico de la pandemia Covid-19, 
considerada como un evento muy estresante, en el consumo de cigarros que se encuentra reflejado en el parámetro significativo relacionado con la variable $\operatorname{Cov} 19_{t}$ e indica que este fenómeno ha ocasionado un incremento importante en la venta de cajetillas de cigarros. Otro aspecto importante, estrechamente vinculado con el anterior, reside en la consecuente restricción a la movilidad de muchas personas, predominantemente en transporte público - en el cuál no es permitido fumar -, ha reforzado el consumo de cigarrillos entre los mexicanos que permanecen en sus casas como un mecanismo de relajamiento (INSP-INPRFM, 2011). El comportamiento miope de los consumidores de cigarros implica que no consideren en sus decisiones los efectos futuros de fumar en su salud, pero que puedan modificar su adición a los cigarros solo por cambios en los precios o en sus ingresos (INSP-INPRFM, 2011).

De acuerdo con todo lo señalado podemos concluir que la pandemia de la Covid-19 y las restricciones a la movilidad de las personas provocaron un aumento temporal del consumo adictivo de cigarros durante la pandemia, que seguramente terminará en el momento que se eliminen las restricciones. El aumento de los precios relativos de los cigarros previo a las condiciones de la pandemia en el primer trimestre y el choque de ingreso a partir del segundo trimestre son la principal causa de la reducción de las ventas de cajetillas de cigarros durante la crisis sanitaria y económica de México en el 2020. La combinación de ambos, en la que ha predominado el efecto de la caída en el ingreso y el aumento en los precios, ha ocasionado el descenso en el consumo de cajetillas de cigarros en promedio, ello no quiere decir que la intensidad en el consumo de cigarros por algunas personas no haya aumentado, seguramente por razones familiares o sociales y en otros por el estrés por el aislamiento social.

\section{Agradecimientos}

Agradecemos a los colegas y estudiantes del Seminario de Economía Urbana y Regional de la UNAM por sus comentarios y observaciones a las versiones previas de esta investigación; a los árbitros de la revista que con sus recomendaciones mejoraron los principales mensajes de la investigación.

\section{Referencias}

[1] Adenji, F. (2019). Consumption function and price elasticity of tobacco demand in Nigeria. Tobacco Prevention and Cessation, 5(48), 1 - 10. doi:https://doi.org/10.18332/tpc/114084

[2] Afonso, J. (2013). Intregrated Modified OLS estimation of cointegrating regression with deterministically trending integrated regressors and residual-based tests for cointegration. En M. Ramos, \& M. Miranda, Estudios en Finanzas y Contabilidad: España y América Latina. Estado del arte y las nuevas metodologías aplicadas (págs. 29 - 86). España: ECORFAN-Madrid.

[3] Apple. (2021). Mapas informes de tendencias de movilidad. doi:https://doi.org/https://covid19.apple.com/mobility

[4] Baltagi, B. H. (2007). On the Use of Panel Data Methods to Estimate Rational Addiction Models. Scottish Journal of Political Economy, 154(1), 1 - 18. doi:https://doi.org/10.1111/j.1467-9485.2007.00402.x 
[5] Bardsley, P., \& Olekalns, N. (1999). Cigarette and Tobacco Consumption: Have Anti-Smoking Policies Made a Difference? The Economic Record, 75(230), 225 - 240. doi:https://doi.org/10.1111/j.14754932.1999.tb02452.x

[6] Becker, G., \& Murphy, K. M. (1988). A Theory of Rational Addiction. Journal of Political economy, 96(4), 675 - 700. Obtenido de https://www.jstor.org/stable/1830469

[7] Becker, G., Grossman, M., \& Murphy, K. M. (1991). Rational Addiction and the Effect of Price on Consumption. The American Economic Review, 81(2), 237 - 241. doi:https://doi.org/0.2307/2006861

[8] Becker, G., Grossman, M., \& Murphy, K. M. (1994). An Empirical Analysis of Cigarette Addiction. The American Economic Review, 81(2), 396 - 418. Obtenido de https://www.jstor.org/stable/2118059

[9] Ben Lakhdar, C., \& Bastianic, T. (2011). Economic constraint and modes of consumption of addictive goods. International Journal of drug Policy, 22(5), 360 - 365. doi:https://doi.org/10.1016/j.drugpo.2011.03.004

[10] CEFP. (2018). El tabaquismo y su efecto en las finanzas públicas: 2007 - 2017. Nota informativa. Obtenido de https://www.cefp.gob.mx/publicaciones/nota/2018/notacefp0042018.pdf

[11] Clarke, H. (2000). Addictive Consumption under Conditions of Risk. The Economic Record, 76(234), $263-272$.

[12] Escario, J. J., \& Molina, J. A. (2020). Why European Consumers Smoke? Responses from the Rational Addiction Model. International Journal of Consumer Studies, 25(1), 24 - 29.

[13] Ferguson, B. (2000). Interpreting the Rational Addiction Model. Health Economics(9), 587 - 598.

[14] Gerrero, C. M., Muñoz, J. A., Sáenz de Miera, B., \& Reynales, L. M. (2013). Consumo de tabaco, mortalidad y política fiscal en México. salud Pública de México, 55(suplemento 2), S276 - S281.

[15] Gjika, A., Zhllima, E., Rama, K., \& Imami, D. (2020). Analysis of Tobacco Price Elasticity in Albania Using Household Level Data. International Journal of Environmental Research and Public Health, 17(2), 1 11.

[16] Grossman, M., \& Chaloupka, F. J. (1998). The demand for cocaine by young adults: a rational addiction approach. Journal of Health Economics(17), 427 - 474.

[17] Guindon, E. G., Paraje, G. R., \& Chaloupka, F. J. (2018). The Impact of Prices and Taxes on the Use of Tobacco Products in Latin America and the Caribbean. American Journal of Public Health(108(S6)), S492 - S502.

[18] Hall, M., Fleischer, N., Reynales, L. M., Arillo, E., \& Trasher, J. (2015). Increasing availability and consumption of single cigarettes: trends and implications for smoking cessation from the ITC Mexico Survey. Tobbaco Control(24), 64 - 70.

[19] INEGI-INSP. (2018). Encuesta Nacional de Salud y Nutrición.

[20] INSP-INPRFM. (2011). Encuesta Nacional de Adicciones.

[21] Iwasaki, N., Tremblay, V., \& Horton, C. (2006). Advertising Restrictions and Cigarette Smoking: Evidence from Myopic and Rational Addiction Models. Contemporary Economic Policy, 24(3), 370 381.

[22] Jiménez, J. A., Sáenz de Miera, B., Reynales, L. M., Water, H. R., \& Hernández, M. (2008). The impact of taxation on tobacco consumption in Mexico. Tobacco Control, 17(2), 105 -110.

[23] Laporte, A., Rohi, A., \& Ferguson, B. S. (2017). Is the Rational Addiction model inherently impossible to estimate? Journal of Health Economics(54), 161 - 175.

[24] Liu, H., Rizzo, J. A., \& Sun, Q. (2015). How do smokers respond to cigarette taxes? Evidence from China's cigarette industry. Health Economics(24), 1314 - 1330.

[25] Mas-Colell, A., Whinston, M. D., \& Green, J. R. (1995). Microeconomic theory. Oxford University press.

[26] NCI. (2017). A Socioecological Approach to Addressing Tobacco-Related Health Disparities. USA. 
[27] Olivera, R. I., Cermeño, R., Sáenz de Miera, B., Jiménez, J. A., \& Reynales, L. M. (2010). The effect of tobacco prices on consumption: a time series data analysis for Mexico. Salud Pública de México, 52(S2), S197 - S205.

[28] Phillips, P. C., \& Hansen, B. (1990). Statistical Inference in Instrumental Variables Regression with I(1) Processes. The Review of Economic Studies(1), 99 - 125.

[29] Spanos, A. (2003). Probability Theory and Statistical Inference: Econometric Modeling with Observational Data. London: Cambridge University Press.

[30] Vogelsang, T. J., \& Wagner, M. (2014). Integrated modified OLS estimation and fixed-b inference for cointegrating regressions. Journal of Econometrics, 178(2), 741 - 760.

[31] Wagner, M., \& Wied, D. (2015). Monitoring Stationary and Cointegration. German Economic Association.

[32] WB. (2019). Manual sobre los aspectos económicos de la aplicación de impuestos al tabaco. WB Group.

[33] WHO. (2019). WHO Global report on trends in prevalence of tobacco use 2000 - 2025. WHO Tobacco Control Papers. 